\title{
A New Locally Adaptive Patch Variation Based CT Image Denoising
}

\author{
Manoj Kumar \\ Department of Computer Science Babasaheb Bhimrao Ambedkar University Lucknow, India \\ E-mail: mkjnuiitr@gmail.com \\ Manoj Diwakar \\ Department of Computer Science Babasaheb Bhimrao Ambedkar University Lucknow, India \\ E-mail: manoj.diwakar@gmail.com
}

\begin{abstract}
The main aim of image denoising is to improve the visual quality in terms of edges and textures of images. In Computed Tomography (CT), images are generated with a combination of hardware, software and radiation dose. Generally, CT images are noisy due to hardware/software fault or mathematical computation error or low radiation dose. The analysis and extraction of medical relevant information from noisy CT images are challenging tasks for diagnosing problems. This paper presents a novel edge preserving image denoising technique based on wavelet transform.

The proposed scheme is divided into two phases. In first phase, input CT image is separately denoised using different patch size where denoising is performed based on thresholding and its method noise thresholding. The outcome of first phase provides more than one denoised images. In second phase, block wise variation based aggregation is performed in wavelet domain.

The final outcomes of proposed scheme are excellent in terms of noise suppression and structure preservation. The proposed scheme is compared with existing methods and it is observed that performance of proposed method is superior to existing methods in terms of visual quality, PSNR and Image Quality Index (IQI).
\end{abstract}

Index Terms - Image denoising, Thresholding, Method noise, Correlation analysis, Aggregation.

\section{INTRODUCTION}

Computed Tomography (CT) is an important tool to detect the internal problems in human body such as depiction of lesions in human organs, skull fracture, brain hemorrhage and many more. CT scan images provide extraordinary sensitive medical relevant information which may helpful to diagnose the problems. However, high radiation dose, low contrast, noise and artifacts are some major drawbacks in computed tomography. Still, it is widely used because of low cost, easy availability, short execution time and excellent sharp images. The impact of radiation dose is directly related to CT image quality. High radiation dose may improve the image quality but it may harm to patients. Low radiation dose may degrade the image quality in terms of noise. Due to noisy CT images, the experts may not comfortable to diagnose the problems.

Various techniques have been investigated to control the noise in CT scan imaging. Projection based techniques such as projection space denoising with bilateral filtering and $\mathrm{CT}$ noise modeling for dose reduction in $\mathrm{CT}$ imaging [1] work on raw data or sinogram, where noise filtering is performed on raw data or sinogram and the denoised image is reconstructed. Many iterative reconstruction approaches for noise suppression in CT have also been proposed such as ordered subset based reconstruction for X-ray CT scan [2] optimizes the statistical functions and improve low dose CT images. Most of other techniques e.g., bilateral filtering [1, 3], total variation denoising $[4,5]$, nonlocal means (NLM) denoising [6, 7] and k-SVD [8] take the advantages of statistical properties of objects in image space and preserve clinical structures such as sharp edges, similarities between neighboring pixels, etc. In transformdomain denoising techniques, the input data is decomposed into its scale-space representation [9]. Linear filters such as Wiener filter [10] in the wavelet domain give optimal results when the signal distortion is estimated as Gaussian approximation and the accuracy is measured by calculating Mean Square Error (MSE). Various thresholding techniques for noise reduction have also been introduced with wavelet transform such as efficient image denoising method based on a new adaptive wavelet packet thresholding function [11, 12], ideal spatial adaptation via wavelet shrinkage $[13,14]$ etc. Thresholding is one of the important tools for denoising. VISUShrink [13, 15] is a non-adaptive universal threshold, which depends only on number of samples and known to find smoothed images because its threshold choice can be large due to its dependence on the number of pixels in the images. SUREShrink [13, 16] uses a hybrid of the universal and the SURE [Steins Unbiased Risk Estimator] thresholds, and performs better than VISUShrink. BayesShrink [17-19] minimizes the Bayes risk estimator function assuming generalized Gaussian approximation and thus finds adaptive threshold value. Chang et al. [20] proposed the use of adaptive wavelet thresholding for image denoising, by modeling the 
wavelet coefficients as a generalized Gaussian random variable, whose parameters are estimated locally (i.e., within a given neighborhood). In image space, CT images can be denoised directly without access to raw data. CT image denoising is a challenging task because of finding correct noise variation, relationship between coefficients and achieving an optimal trade-off between denoising and blurring or artifacts [21-23]. To surmount these challenges, we propose a maximum edge preserving and noise reduction method. Experimentally, it is observed that each wavelet coefficients are affected with different patch size. With different patch size, the estimated threshold value may not be the same and may affect images in terms of local features and noise. With this consideration, we propose a scheme for CT image denoising based on the variation in multiple thresholded values which are obtained by different patch sizes.

The paper is organized as follows. Section 2 gives a brief overview of wavelet based shrinkage. In section 3 , the proposed method is presented in details. Experimental results, including discussions and comparison with other denoising methods, are given in section 4. Finally, conclusions are summarized in section 5 .

\section{IMAGE DENOISING BY WAVELET SHRINKAGE}

Wavelet transform is a powerful tool for signal and image processing tasks because of multi-resolution analysis, sub-banding and localized in both, frequency and time domain. Two dimensional DWT transforms the signals into low frequency (LL) and high frequency components (LH; HL; HH), as shown in Fig. 1.

With the assumption, the CT images are corrupted by Gaussian noise with zero mean and different variances, the noisy CT image can be expressed as:

$$
X(m, n)=Y(m, n)+\eta(m, n)
$$

Where, $\eta(m, n)$ is a noise coefficient, $Y(m, n)$ and $X(m, n)$ are noiseless and noisy images respectively.

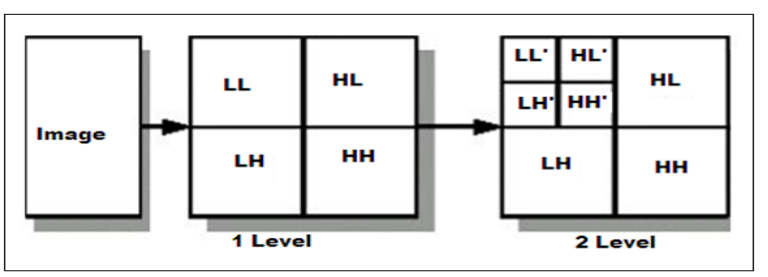

(a)

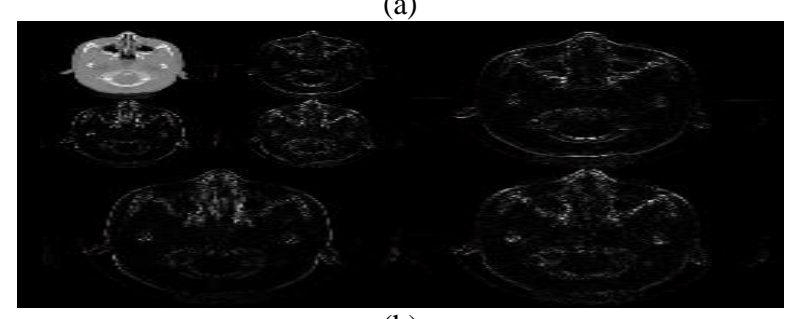

(b)

Fig.1. (a) First and Second Level 2-D Wavelet Decomposition (b) 2DDWT Decomposition on CT image up-to two levels.
Before applying wavelet thresholding, two important tasks must be performed which are responsible for the results and computational complexity. First, a wavelet basis is chosen for determining its decomposing layers such as haar, db2, etc. Second, decomposition level is chosen for thresholding on all subbands for all levels. Wavelet based noise shrinkage can be expressed with the following major steps:

Step 1: Perform discrete wavelet transform (DWT) on input noisy $\mathrm{CT}$ image to obtain approximation and detail parts.

Step 2: Perform the denoising using following steps:

i. Estimate noise variance

ii. Apply thresholding on detail parts

Step 3: Apply inverse discrete wavelet transform (Inverse DWT) to obtain final denoised image.

\section{A. Threshold Selection}

For CT images, selection of a threshold value is not an easy task. By selecting small threshold value, the resultant image may be noisy. For large threshold value, the resultant image may blur on the edges. The selection of optimal threshold value is necessary and important task for preserving clinical details and suppression of noise.

The threshold $\lambda$ can be selected as:

$$
\lambda=\left(\frac{\sigma_{\eta}^{2}}{\sigma_{Y}}\right)
$$

Where the noise variance can be estimated using robust median estimation method (Abramovitch et al. 1998) as follows:

$$
\sigma_{\eta}^{2}=\left[\frac{\text { median }(|X(m, n)|)}{0.6745}\right]^{2},
$$

Where, $X(m, n) \in H H_{L}, \mathrm{~L}$ represents respective level in wavelet decomposition. The standard deviation of noise less image $\left(\sigma_{Y}\right)$ can be estimated as:

$$
\sigma_{Y}^{2}=\max \left(\sigma_{X}^{2}-\sigma_{\eta}^{2}, 0\right)
$$

Where, $\sigma_{X}^{2}=\frac{1}{b} \sum_{i=1}^{b} X_{i}^{2}$, and $b$ represent patch size of an input image.

\section{B. Threshoding process}

After selecting a threshold value, the process of 
thresholding is applied by selecting an appropriate algorithm. Hard thresholding and soft thresholding methods are very popular for thresholding. In hard thresholding, each coefficient value is compared with threshold value and values less than threshold are replaced by zero. In Soft thresholding, replacement process is same as in hard thresholding, additionally rest of coefficients are modified by subtracting threshold values. In comparison of both, Soft thresholding gives better performance for visual appearance of images. The soft thresholding can be expressed as:

$$
\hat{Y}:=\left\{\begin{array}{cc}
0 & \text { if }|X| \leq \lambda \\
\operatorname{sign}(X)(|X|-\lambda) & \text { if }|X|>\lambda
\end{array}\right.
$$

\section{PRoposed Methodology}

The perfect optimization for estimating a threshold value is an almost impossible task for image denoising. Estimated threshold value may be varying according to different patch sizes. With the consideration that the small variation of neighborhood size will not much affect the threshold estimated value, a new locally adaptive different patch size based thresholding scheme is proposed, where some limited patch sizes are selected. The patch sizes can be extracted as: If $X(m, n)$ is the size of image, then for high frequency subband's first patch size will be $(m / 2, n / 2)$. Similarly, $k-1$ numbers of subband are generated by dividing previous patch size by 2 .

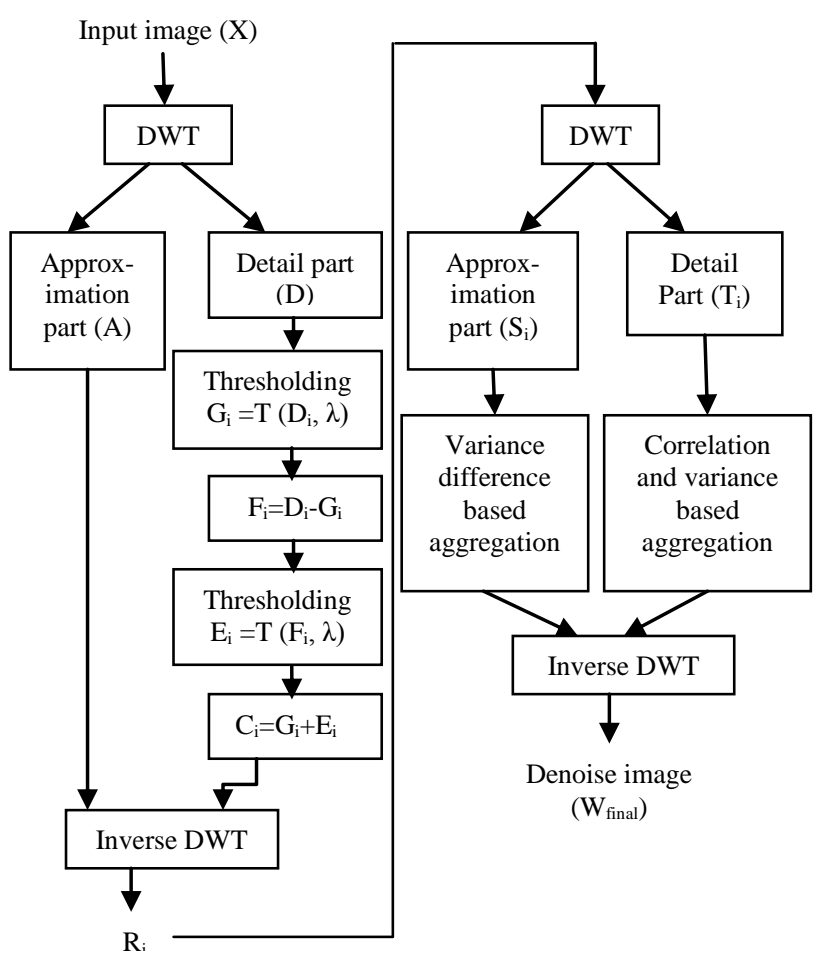

Fig.2. Flow Chart of Proposed Scheme

With the assumption, that the CT image is corrupted by
Gaussian noise, the proposed method as shown in fig. 2 can be summarized as:

1) Select the maximum size of input noisy CT image $X(m, n)$ where $m=2^{k}$ and $n=2^{k}$.

2) Apply DWT on input image to obtain low frequency ( $A$ ) and high frequency ( $D$ ) subbands.

3) High frequency subbands $\left(D_{i}\right)$ are patching wise threshold (for $i=1$ to $k-1$ ) using equation (5) according to respective patch size.

$$
G_{i}=T\left(D_{i}, \lambda\right)
$$

Where, $\lambda$ is an estimated noise level calculated using equation (3).

4) Apply method noise using input high frequency ( $D$ ) subband and thresholded high frequency subband $\left(G_{i}\right)$ as:

i) Subtract $G_{i}$ from $D$ and get $F_{i}$ as,

$$
F_{i}=D-G_{i}
$$

ii) Apply patch wise thresholding on $F_{i}$.

$$
E_{i}=T\left(F_{i}, \lambda\right)
$$

iii) Add $G_{i}$ and $E_{i}$ and get $C_{i}$,

$$
C_{i}=G_{i}+E_{i}
$$

5) Apply inverse DWT, using low frequency ( $A$ ) and thresholded high frequency $\left(C_{i}\right)$ subbands. The output $\left(R_{i}\right)$ comes in the form of $k-1$ denoised images.

6) To obtain final denoised image, perform the following steps:

i) Separately apply DWT on $R_{i}$ images to obtain their approximation $\left(S_{i}\right)$ and detail parts $\left(T_{i}\right)$.

ii) Estimate the variance at each pixel of approximation part at level 2 of all denoise images $\left(R_{i}\right)$ in block of size $3 \times 3$.

iii) Variance (Var) difference on approximation part of denoised images ( $R_{i}$ ), as below:

$$
\tau=\frac{1}{k-2} \sum_{j=1}^{k-2} \alpha_{j}
$$

where, 


$$
\alpha_{j}=\operatorname{Var}\left(S_{j}\right)-\operatorname{Var}\left(S_{j+1}\right), j=1 \text { to } k-2
$$

The value of $\tau$ is normalized in the range [0 1 1] and used as a weight factor for the next process.

iv) Variance difference based aggregation process is based on weight factors $\tau$ and perform on approximation part as per the following relationship:

$$
B_{\text {final }}=H+Q
$$

where,

$$
H=\frac{1}{k-1} \sum_{f=1}^{\frac{k-1}{2}} \frac{\tau}{f}\left(S_{f}\right) ; \quad Q=\frac{1}{k-1} \sum_{g=\frac{k-1}{2}+1}^{k-1}\left(1-\left(\frac{\tau}{(k-g)}\right)\right)\left(S_{g}\right)
$$

v) Variance based aggregation (Jain et al. 2015) using correlation (Corr) is performed on detail parts using following relationship:

$$
C_{\text {final }}= \begin{cases}T_{1}^{b}, & \text { If } \operatorname{Corr}\left(\mathrm{T}_{\mathrm{i}}, \mathrm{T}_{\mathrm{i}+1}\right)>\mathrm{Th} \\ \sum_{i=1}^{k-1} \beta^{b} T_{i}^{b}, & \text { Otherwise }\end{cases}
$$

where,

$$
\beta^{b}=\frac{\operatorname{Var}^{-1}\left(T_{1}^{b}\right)}{\sum_{i=1}^{k-1} \operatorname{Var}^{-1}\left(T_{i}^{b}\right)}
$$

$T_{i}$ represents detail part of all denoised images $\left(R_{i}\right)$ with block size $b . T_{h}$ represents a defined threshold value to differentiate between correlated and uncorrelated coefficients. The function $\operatorname{Var}^{-1}$ (.) can be estimated as inverse variance.

\section{7) Apply inverse DWT using $B_{\text {final }}$ and $C_{\text {final }}$ to obtain final denoised image $\left(W_{\text {final }}\right)$.}

In proposed scheme, noisy CT image is denoised in wavelet domain where noise on high frequency coefficients of each subband in $L$ level has been suppressed using Bayes shrink and method noise thresholding with different patch sizes. In method noise, the thresholded patch is subtracted with original patch. The outcome of subtraction is again thresholded and added into the thresholded original patch. This method noise thresholding process is used to observe, how much local features of clinical details are missing at the time of thresholding and can be recovered as shown in Step 4 . With different patch size, separate multiple denoised images are obtained. To get final denoised image, separately DWT is performed on all denoised images. In approximation part of all denoised images, a weight value $\tau$ is estimated using variance difference between different denoised images. This weight value is incorporated into denoised images and aggregation relationship has been performed as shown in Step 6(iv). In detail part, patch wise correlation coefficient values are obtained between all denoised images. Correlation value lies in the interval $[-1 ; 1]$, where 1 means perfect correlation, 0 no correlation, and -1 perfect anticorrelation. To find correlated and uncorrelated values, correlation values are normalized in the range of $[0 ; 1]$. The correlation values closer to 1 indicate that the similarity structure is present between the images. The lower values (near to 0 ) indicate that the corresponding value includes only noise and, therefore, can be suppressed. If correlation value is greater with a defined threshold $\left(T_{h}\right)$ value then keep the largest patch size denoised coefficient value of subband, otherwise, perform variance based weighted average between all denoised coefficients of subband as given in Step 6(v). Thus, final denoised CT image can be obtained using inverse wavelet transform.

\section{EXPERIMENTAL RESULTS}

The experimental evaluation is performed on low quality CT images with size $512 \times 512$. The CT scanned test images shown in fig.3(a-c) is obtained from public access database(https://eddie.via.cornell.edu/cgibin/datac/logon. cgi), and CT scanned test image shown in fig. 3(d) is obtained from a Diagnosis Center. The proposed image denoising method is applied on all test images corrupted by additive Gaussian white noise at six different noise level $(\sigma)$ : 10, 15, 20, 25, 30, and 35. Fig. 3(a), 3(b), 3(c) and 3(d) are considered as CT images 1, 2, 3 and 4, respectively. Fig. 4(a-d) are showing noisy test image data set with $\sigma=25$.

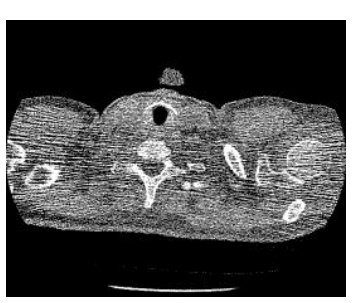

(a) CT 1 image

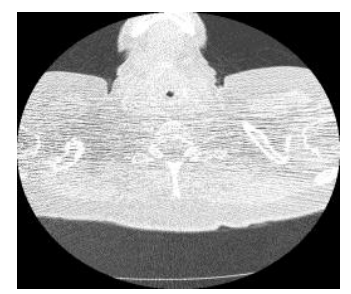

(c) CT 3 image

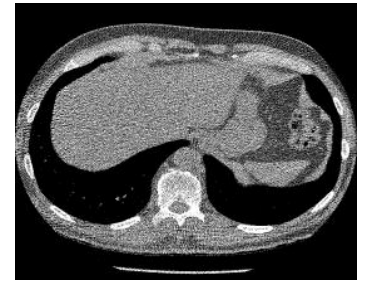

(b) CT 2 image

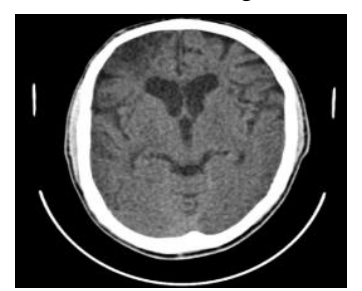

(d) CT 4 image
Fig.3. Input Test CT Image Dataset 


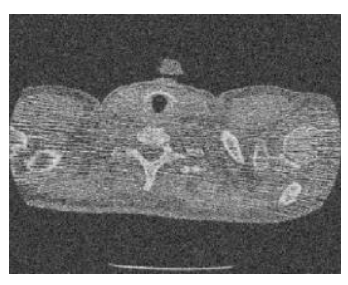

(a) CT 1 image

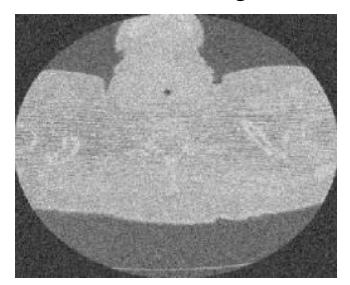

(c) CT 3 image

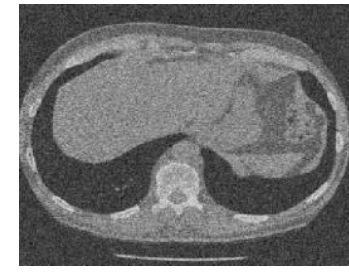

(b) CT 2 image

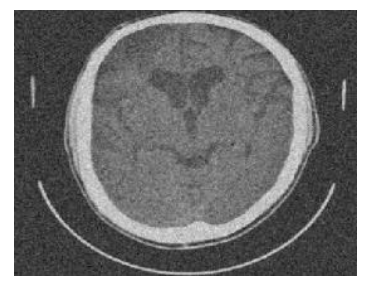

(d) CT 4 image
Fig.4. Noisy CT Image Dataset $(\sigma=25)$

\section{A. Evaluation of different patch size}

$k-1$ numbers of patch sizes are used to denoise the images using proposed methodology. The outcome of thresholding may be differ with differ local patch sizes. As $k-1$ numbers of patch size are used separately for thresholding, $k-1$ numbers of thresholded subband are obtained respectively. To verify the effect of different patch sizes for thresholding, an average correlation value is obtained between $k-1$ thresholded subband for all test images. Table 1 shows the average values of the correlations using a $7 \times 7$ window for all $\mathrm{CT}$ image dataset.

Table 1. Average Values of the Correlations between K-1 Denoised Images using Different Patch Size

\begin{tabular}{|c|c|c|c|c|}
\hline Level $(L)$ & $L=1$ & $L=2$ & $L=3$ & $L=4$ \\
\hline CT 1 image & 0.9501 & 0.9385 & 0.8976 & 0.8793 \\
\hline CT 2 image & 0.9729 & 0.9462 & 0.8991 & 0.8592 \\
\hline CT 3 image & 0.9201 & 0.8976 & 0.8715 & 0.8358 \\
\hline CT 4 image & 0.8973 & 0.8695 & 0.8413 & 0.8187 \\
\hline
\end{tabular}

\section{B. Performance evaluation}

Peak Signal-to-noise Ratio (PSNR) is an important factor to evaluate denoising performance. The high PSNR value represents more similarity between the denoising and original image than lower PSNR value. The objective quality of the denoised image is measured by PSNR as:

$$
P S N R=10 \log _{10} \frac{255^{2}}{m s e} \mathrm{~dB}
$$

Where mse is the mean square error between the original and the denoised image:

$$
m s e=\frac{1}{m n} \sum_{i=1}^{m} \sum_{j=1}^{n}[X(i, j)-W(i, j)]^{2}
$$

Image quality index (IQI) is another important factor to analyse the performance of image denoising in terms of correlation, luminance distortion and contrast distortion. For input image (X) and denoised image (W), the IQI can be defined as:

$$
I Q I=\frac{4 \sigma_{X W} \bar{X} \bar{W}}{\left(\sigma_{X}^{2}+\sigma_{W}^{2}\right)\left[(\bar{X})^{2}+(\bar{W})^{2}\right]}
$$

where,

$$
\begin{aligned}
\bar{X}=\frac{1}{N} \sum_{i=1}^{N} X_{i}, \bar{W} & =\frac{1}{N} \sum_{i=1}^{N} W_{i}, \sigma_{X}^{2}=\frac{1}{N-1} \sum_{i=1}^{N}\left(X_{i}-\bar{X}\right)^{2}, \\
\sigma_{W}^{2} & =\frac{1}{N-1} \sum_{i=1}^{N}\left(W_{i}-\bar{W}\right)^{2}
\end{aligned}
$$

and

$$
\sigma_{X W}=\frac{1}{N-1} \sum_{i=1}^{N}\left(X_{i}-\bar{X}\right)\left(W_{i}-\bar{W}\right)
$$

The quality of image index range lies between 1 and -1 . The highest value 1 represents an identical value of input image pixel and denoised image pixel. The lowest value 1 shows that the pixels values are uncorrelated.

\section{Experimental evaluation}

To evaluate the results of proposed scheme, the parameters are kept fixed throughout the comparisons as in other methods. The result evaluation is performed on the CT images with the size of $512 \times 512$, can be expressed as $2^{9} \times 2^{9}$. As per proposed scheme, we can evaluate the total number of patch sizes are k-1 i.e 8 and patch size can be expressed as: 256x256, 128x128, 64x64, 32x32, $16 \times 16,8 \times 8,4 \times 4$ and $2 \times 2$. Using these patch sizes, 8 denoised images are obtained. The method noise helps to recover details, textures and edges from denoised images which represent the performance and limitations of denoising algorithm. It is observed from the experimental results that too small blocks may affect the denoised image as block artifacts and too large block size may affect the details as a poor quality edges. Therefore, we apply aggregation process to get final denoised image using multiple denoised images. To aggregate the denoisied images, correlation values are measured on high frequency subbands using block size $3 \times 3$ for all test images where a threshold value $\left(T_{h}\right)$ is set as 0.96 . Above the threshold value is considered as similarity, so large patch size of denoised coefficients are opted. For below threshold value, variation based weighted average is used with block size $3 \times 3$. Similarly, variance difference based aggregation is performed on low frequency subband. Final output comes in the form of single denoised image. 


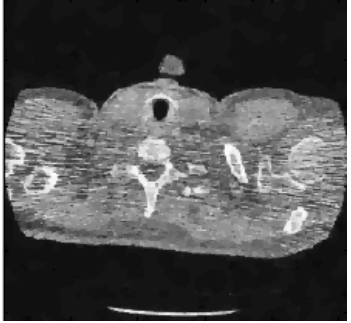

(a) CT 1 image

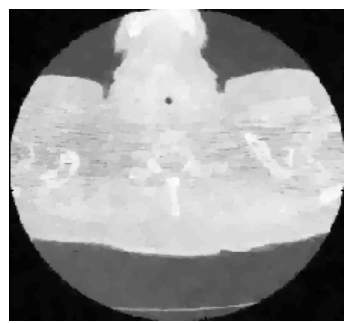

(c) CT 3 image

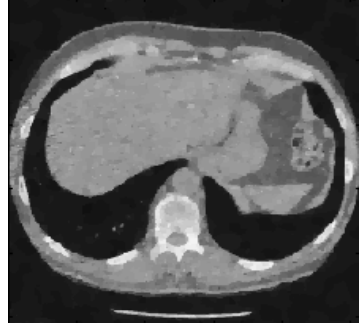

(b) CT 2 image

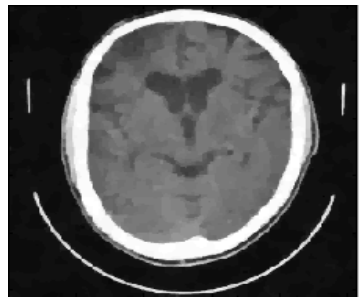

(d) CT 4 image

Fig.5. Results of Total Variation Denoising

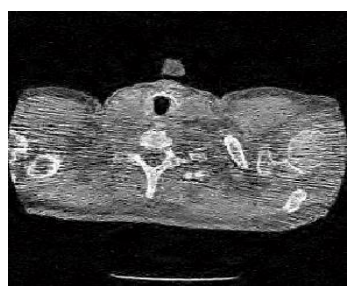

(a) CT 1 image

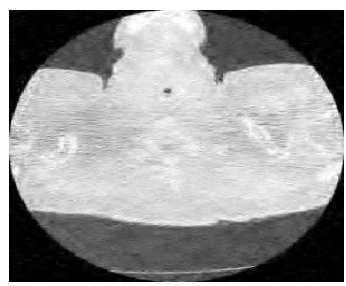

(c) CT 3 image

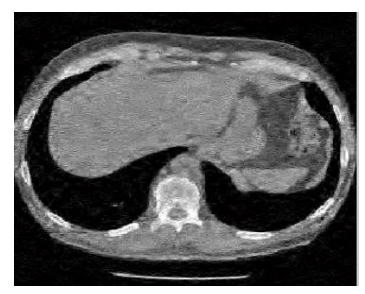

(b) CT 2 image

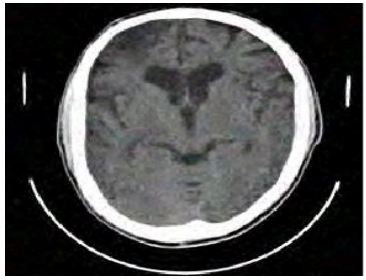

(d) CT 4 image

Fig.6. Results of SURELET

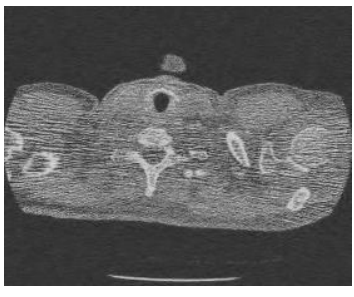

(a) CT 1 image

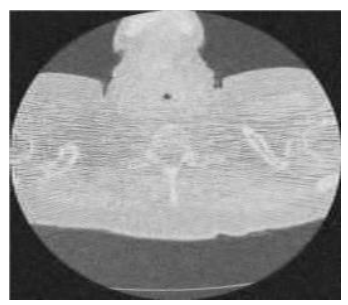

(c) CT 3 image

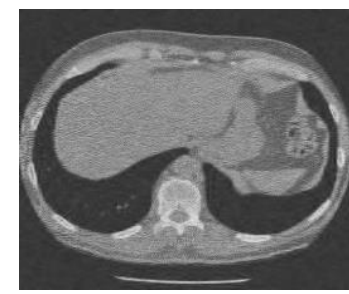

(b) CT 2 image

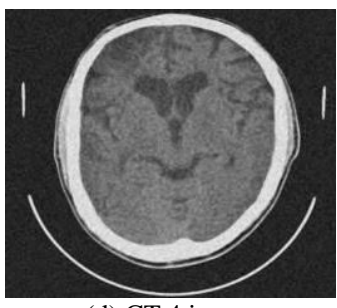

(d) CT 4 image

Fig.7. Results of Bayes Thresholding

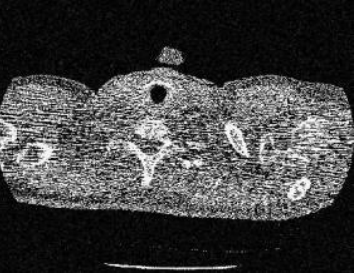

(a) CT 1 image

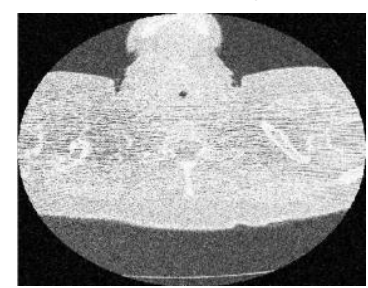

(c) CT 3 image

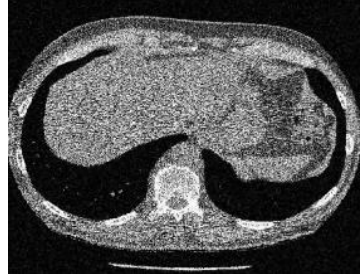

(b) CT 2 image

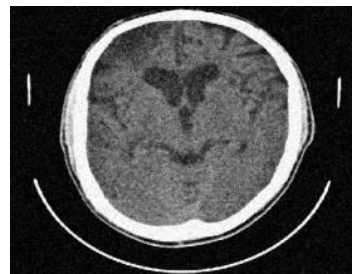

(d) CT 4 image
Fig.8. Results of Proposed Scheme

Table 2. PSNR of Denoised Images

\begin{tabular}{|c|c|c|c|c|c|}
\hline & $\sigma$ & $\begin{array}{c}\text { TV } \\
\text { method }\end{array}$ & $\begin{array}{l}\text { SURELE } \\
\text { T method }\end{array}$ & $\begin{array}{l}\text { Bayes } \\
\text { method }\end{array}$ & $\begin{array}{c}\text { Proposed } \\
\text { method }\end{array}$ \\
\hline \multirow{6}{*}{$\begin{array}{c}\text { CT } 1 \\
\text { image }\end{array}$} & 10 & 32.14 & 33.25 & 31.50 & 33.39 \\
\hline & 15 & 30.95 & 31.45 & 29.96 & 31.44 \\
\hline & 20 & 29.45 & 30.10 & 28.21 & 30.05 \\
\hline & 25 & 27.98 & 29.68 & 28.01 & 29.85 \\
\hline & 30 & 26.31 & 28.47 & 27.25 & 28.54 \\
\hline & 35 & 25.26 & 26.19 & 25.31 & 26.88 \\
\hline \multirow{6}{*}{$\begin{array}{l}\text { CT } 2 \\
\text { image }\end{array}$} & 10 & 31.54 & 32.12 & 30.98 & 32.47 \\
\hline & 15 & 30.87 & 30.64 & 29.42 & 31.05 \\
\hline & 20 & 28.95 & 29.08 & 28.47 & 29.53 \\
\hline & 25 & 28.48 & 28.64 & 27.26 & 28.96 \\
\hline & 30 & 27.69 & 28.03 & 26.17 & 28.11 \\
\hline & 35 & 25.83 & 26.96 & 25.34 & 26.97 \\
\hline \multirow{6}{*}{$\begin{array}{l}\text { CT } 3 \\
\text { image }\end{array}$} & 10 & 32.33 & 33.19 & 31.98 & 33.89 \\
\hline & 15 & 31.29 & 31.25 & 30.67 & 31.87 \\
\hline & 20 & 29.84 & 30.98 & 28.68 & 30.91 \\
\hline & 25 & 27.15 & 29.27 & 28.34 & 29.31 \\
\hline & 30 & 26.29 & 28.54 & 27.52 & 28.67 \\
\hline & 35 & 24.36 & 26.65 & 24.64 & 26.73 \\
\hline \multirow{6}{*}{$\begin{array}{c}\text { CT } 4 \\
\text { image }\end{array}$} & 10 & 32.65 & 33.65 & 31.63 & 33.79 \\
\hline & 15 & 31.35 & 31.24 & 29.26 & 31.35 \\
\hline & 20 & 29.64 & 30.19 & 28.31 & 30.61 \\
\hline & 25 & 27.45 & 29.34 & 28.72 & 29.36 \\
\hline & 30 & 26.64 & 28.21 & 27.37 & 28.42 \\
\hline & 35 & 25.39 & 26.94 & 25.61 & 26.61 \\
\hline
\end{tabular}

\section{Comparisons}

To validate the superiority of the proposed scheme, its performance is compared in terms of visual quality, PSNR, and Image Quality Index (IQI) of the denoised images using the various methods available in literature such as Total Variation (TV) (Goldstein et al. 2009), SURELET (Donoho et al. 1995) and Bayes methods (Abramovitch et al. 1998). In all the cases, db8 is used for wavelet decomposition and soft-thresholding is used to 
threshold wavelet coefficients. In WT-based thresholding and proposed scheme, three levels of decomposition are used. Tables $2 \& 3$, respectively show the PSNR (in $\mathrm{dB}$ ) and IQI values of the denoised images relative to their original images using proposed and existing methods.

The best values amongst all the methods are represented in bold. The results shown in tables demonstrate that in most of the cases, the proposed method is superior to all other methods. The results of TV denoising method as shown in fig. 5 gives edge preserved smooth denoised image but texture is not as good as for clinical purpose.

The results of wavelet based denoising using Bayes and SURELET are shown in fig. 6 \& 7 respectively. Both methods are providing smoother results in homogenous regions. As the noise level increases, Bayes method fail to provide smoothed data over the homogenous regions and SURELET also fail to provide satisfactory results on the edges. To overcome these limitations of other methods, the proposed scheme is prepared, where multi estimated threshold values are obtained through different patch sizes, method noise is applied, k-1 denoised images are obtained and finally a aggregation process is applied to provide a single edge preserved noise suppressed image. The obtained results of proposed scheme as shown in fig. 8, gives better results in terms of visual aspects and performance metrics. The result of proposed scheme show improved texture, noise suppression and sharp preserved edges.

Table 3. IQI of Denoised Images

\begin{tabular}{|c|c|c|c|c|c|}
\hline & $\sigma$ & $\begin{array}{c}\text { TV } \\
\text { method }\end{array}$ & $\begin{array}{l}\text { SURELE } \\
\text { T method }\end{array}$ & $\begin{array}{l}\text { Bayes } \\
\text { method }\end{array}$ & $\begin{array}{c}\text { Proposed } \\
\text { method }\end{array}$ \\
\hline \multirow{6}{*}{$\begin{array}{l}\text { CT } 1 \\
\text { image }\end{array}$} & 10 & 0.9931 & 0.9912 & 0.9924 & 0.9976 \\
\hline & 15 & 0.9534 & 0.9856 & 0.9762 & 0.9865 \\
\hline & 20 & 0.9312 & 0.9541 & 0.9365 & 0.9597 \\
\hline & 25 & 0.8972 & 0.9165 & 0.9174 & 0.9248 \\
\hline & 30 & 0.8903 & 0.8954 & 0.8832 & 0.8962 \\
\hline & 35 & 0.8894 & 0.8762 & 0.8614 & 0.8747 \\
\hline \multirow{6}{*}{$\begin{array}{l}\text { CT } 2 \\
\text { image }\end{array}$} & 10 & 0.9817 & 0.9828 & 0.9751 & 0.9889 \\
\hline & 15 & 0.9789 & 0.9794 & 0.9745 & 0.9831 \\
\hline & 20 & 0.9421 & 0.9654 & 0.9241 & 0.9521 \\
\hline & 25 & 0.8452 & 0.8684 & 0.8922 & 0.9047 \\
\hline & 30 & 0.8364 & 0.8361 & 0.8632 & 0.8740 \\
\hline & 35 & 0.8189 & 0.8314 & 0.8614 & 0.8694 \\
\hline \multirow{6}{*}{$\begin{array}{l}\text { CT } 3 \\
\text { image }\end{array}$} & 10 & 0.9874 & 0.9812 & 0.9914 & 0.9965 \\
\hline & 15 & 0.9514 & 0.9614 & 0.9762 & 0.9893 \\
\hline & 20 & 0.9423 & 0.9591 & 0.9432 & 0.9614 \\
\hline & 25 & 0.9102 & 0.9241 & 0.9397 & 0.9235 \\
\hline & 30 & 0.8964 & 0.8931 & 0.8942 & 0.9131 \\
\hline & 35 & 0.8831 & 0.8894 & 0.8913 & 0.8941 \\
\hline \multirow{6}{*}{$\begin{array}{c}\text { CT } 4 \\
\text { image }\end{array}$} & 10 & 0.9871 & 0.9974 & 0.9954 & 0.9979 \\
\hline & 15 & 0.9642 & 0.9831 & 0.9645 & 0.9846 \\
\hline & 20 & 0.9409 & 0.9641 & 0.9469 & 0.9698 \\
\hline & 25 & 0.9123 & 0.9352 & 0.9231 & 0.9411 \\
\hline & 30 & 0.8991 & 0.8978 & 0.8945 & 0.9006 \\
\hline & 35 & 0.8647 & 0.8649 & 0.8791 & 0.8771 \\
\hline
\end{tabular}

\section{CONCLUSIONS}

In this paper, a scheme is proposed based on locally patch thresholding, its method noise thresholding and aggregation. Due to selective patch sizes, the cost computation is reduced in compare of iterative methods.
The proposed scheme is taking the advantage of thresholding to denoised same pixel with different patch size. A gap in patch size is maintained by apply a rule to divide by 2 of previous patch size. A method noise with selective patch size may also help for more denoised and edge preserved patch. An aggregation scheme helps to provide a single final edge preserved and noise suppressed CT image. Final outcomes of proposed scheme are good in terms of noise suppression and edge preservation. All the results of proposed scheme are compared by existing methods. In most of the cases, the performance of proposed scheme is giving better values in terms of PSNR and IQI. Apart from performance metrics, the visual quality of proposed scheme over the CT images is also better in terms of clinically relevant details. Experimental results demonstrate that our proposed scheme: (i) effectively eliminate the noise in CT images, (ii) preserve the edge and structural information, and (iii) retain clinically relevant details.

\section{REFERENCES}

[1] A. Manduca, L. Yu, J. D. Trzasko, N. Khaylova, J. M. Kofler, C. M. McCollough and J. G. Fletcher, "Projection space denoising with bilateral filtering and CT noise modeling for dose reduction in CT," International Journal of Medical Physics Research and Practice, Vol. 36, No. 11, pp.4911-4919, 2009.

[2] D. Kim, S. Ramani and J. A. Fessler," Accelerating X-ray CT ordered subsets image reconstruction with Nesterov's first-order methods" In Proc. Intl. Mtg. on Fully 3D Image Recon. in Rad. and Nuc. Med pp. 22-5, 2013.

[3] F. Durand and J. Dorsey, "Fast bilateral filtering for the display of high dynamic range images," ACM Transactions on Graphics, Vol. 21, No. 3, pp.257-266, 2002.

[4] T. Goldstein and S. Osher, "The Split Bregman Method for L1 Regularized Problems," SIAM Journal on Imaging Sciences, Vol. 2, No. 2, pp.323-34, 2009.

[5] A. Chambolle, "An algorithm for total variation minimization and applications," Journal of Matter Image and Visualization', Journal Roy Statistic Society, Vol. 20, No. 1, pp.89-97, 2004.

[6] A. Buades, B. Coll and J. M. Morel Song, "A review of image denoising algorithms, with a new one," SIAM Journal on Multiscale Modeling and Simulation, Vol. 4, No. 2, pp.490-530, 2005.

[7] Z. Li, L.Yu, J. D. Trzasko, D. S. Lake, D. J. Blezek, J. G. Fletcher, C. H. McCollough and A. Manduca, "Adaptive nonlocal means filtering based on local noise level for CT denoising," International Journal of Medical Physics Research and Practice, Vol. 41, No. 1, 2014.

[8] M. Aharon, M. Elad and A. Bruckstein, "K-SVD: An algorithm for designing overcomplete dictionaries for sparse representation', IEEE Trans. Signal Process, Vol. 54, pp.4311-4322, 2006.

[9] S. Mallat, "A theory for multiresolution signal decomposition: the wavelet representation," IEEE Trans. on Pattern Anal. Mach. Intell. , Vol. 11, No. 7, pp.674693, 1989.

[10] K. Li and R. Zhang, "Multiscale wiener filtering method for low-dose CT images," IEEE Biomedical Engineering and Informatics, pp.428-431, 2010.

[11] A. Fathi and A. R. Naghsh-Nilchi, "Efficient image denoising method based on a new adaptive wavelet packet 
thresholding function," IEEE Trans Image Process, Vol. 21, No. 9, pp.3981-3990, 1989.

[12] M. Malfait and D. Roose, "Wavelet based image denoising using a Markov Random Field a priori model," IEEE Transactions on Image Processing, Vol. 6, No. 4, pp.549-565, 1997.

[13] D. L. Donoho and I. M. Johnstone, "Ideal spatial adaptation via wavelet shrinkage," Biometrika, Vol. 81, pp.425-455, 1994.

[14] A. Borsdorf, R. Raupach, T. Flohr and J. Hornegger Tanaka, "Wavelet Based Noise Reduction in CT-Images Using Correlation Analysis," IEEE Transactions on Medical Imaging, Vol. 27, No. 12, pp.1685-1703, 2008.

[15] M. C. Motwani, M. C. Gadiya, R. C. Motwani and F. C. Harris, "Survey of Image Denoising Techniques," Proceedings of Global Signal Processing Expo and Conference (GSPx '04), pp.27-30, 2004.

[16] D. L. Donoho, "De-noising by soft-thresholding," IEEE Transactions on Information Theory, Vol. 41, No. 3, pp.613-627., Signal Process. Vol. 90 no. 8 pp 2529-2539, 2010, 1995.

[17] P. Moulin and J. Liu "Analysis of multiresolution image denoising schemes using generalized Gaussian and complexity priors," IEEE Infor. Theory, Vol. 45, No. 3, pp.909-919, 1999.

[18] F. Abramovitch, T. Sapatinas, and B. W. Silverman "Wavelet thresholding via a Bayesian approach," Journal Roy Statistic Society, Vol. 60, No. 4, pp.725-749, 1998.

[19] J. Romberg, H. Choi and R. G. Baraniuk, "Bayesian wavelet domain image modeling using hidden Markov models," IEEE Transactions on Image Processing, Vol. 10, pp.1056-1068, 2001.

[20] S. G. Chang, B. Yu and M. Vetterli, "Adaptive wavelet thresholding for image denoising and compression," IEEE Trans. on Image Proc, Vol. 9, No. 9, pp.1532-1546, 2000.

[21] L. Xinhao, M. Tanaka and M. Okutomi, "Single- Image Noise Level Estimation for Blind Denoising," IEEE Transactions on Image Processing, Vol. 22, No. 12, pp.5226-5237, 2013.

[22] H. S. Bhadauria and M. L. Dewal, "Efficient Denoising
Technique for CT images to Enhance Brain Hemorrhage Segmentation," International Journal of Digit Imaging, Vol.25, No. 6, pp. 782-791, 2012.

[23] P. Jain and V. Tyagi, "LAPB: Locally adaptive patchbased wavelet domain edge-preserving image denoising," Journal of Information Sciences, Vol. 294, pp. 164-181, 2015.

\section{Authors' Profiles}

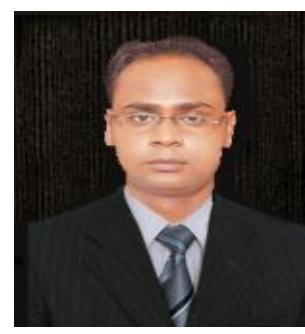

Manoj Kumar: He has achieved his master degree (M. C. A.) from JNU, New Delhi and Ph.D from IIT Roorkee, India. Currently, he is working as Assistant Professor in the Department of Computer Science, Babasaheb Bhimrao Ambedkar University, Lucknow, India. His research interest includes Computer Graphics, Vision, \& Image processing, Medical imaging and Pattern recognition. He has published various research papers in national and international journals \& conferences.

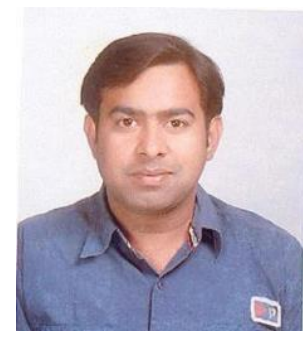

Manoj Diwakar: He has achieved his B.Tech degree from Dr. R. M. L. Awadh University, Faizabad and M.Tech from MITS, Gwalior, India. $\mathrm{He}$ is currently pursuing his $\mathrm{Ph}$. D. in the Department of Computer Science, Babasaheb Bhimrao Ambedkar University, Lucknow, India. His research interests include Image processing, Information Security and Medical imaging. He has published various research papers in national and international journals \& conferences.

How to cite this paper: Manoj Kumar, Manoj Diwakar,"A New Locally Adaptive Patch Variation Based CT Image Denoising", International Journal of Image, Graphics and Signal Processing(IJIGSP), Vol.8, No.1, pp.43-50, 2016.DOI: 10.5815/ijigsp.2016.01.05 